\title{
Energy Monitoring System Incorporating Energy Profiling and Predictive Household Movement for Energy Anomaly Detection
}

\author{
Elmer R. Magsino \\ Department of Electronics and Communications Engineering, \\ Gokongwei College of Engineering, De La Salle University, Manila, Philippines \\ elmer.magsino@dlsu.edu.ph
}

\begin{abstract}
In most buildings and large households, an energy management system is needed to timely monitor and adaptively make decisions regarding the utility of the electrical equipment/appliances in order to minimize energy cost and provide convenience and comfort to the occupants. However, for small households, i.e., with four to five family members, energy monitoring systems are more appropriate since the number of appliances and households are restricted. Also, the household movements are limited, therefore, can be profiled and predicted. In this work, a home energy monitoring system, EnMonSys, with predictive household movement for small and compact houses is designed and implemented to detect common energy anomaly such as the opening/leaving a refrigerator's door open. This study has found out through experiments that factors such as changes in household member and/or season greatly affect the energy consumption, instead of the equipment/appliance control. Currently, EnMonSys has correctly detected $81 \%$ of energy anomaly and is believed to increase as the household profile (movement and appliance usage) is adjusted accordingly
\end{abstract}

Key words: Energy Anomaly, Energy Monitoring System, Household Energy Profile, Predictive Household Movement.

\section{INTRODUCTION}

Internet of Things (IoT) deals with the interconnectivity of devices in an area through wireless connection for attaining specific objectives such as data gathering, environment monitoring, and improving daily processes. [1], [2]. The abundance of commercially available and inexpensive sensor devices and communications modules have taken the IoT paradigm to a new level of different applications. IoT has been widely employed in information sharing among vehicles and roadside units [3], in health care utilizing body sensor networks monitoring human body functions [4], in personal and social domains concerning social networking, theft and losses [5], and smart infrastructures such as houses [6], buildings [7], and industrial equipment [8].
In smart homes, many household states e.g., light status, air conditioning temperature, etc., are monitored to provide the necessary and corresponding actuation to achieve an optimization goal, e.g., energy efficiency, energy cost, home automation, security, and living comfort and convenience. In [9], a holistic approach of a smart home was presented. It tackled the various IoT components, practical IoT framework design, and data processing needed in a smart home. A simple home automation example was discussed in [10], where common home appliances are turned ON or OFF when the owner or a guest is approaching. In [11], the network-level security and privacy of IoT devices were studied. Aware of the real threats of intrusion from unwanted attacks, security solutions were proposed to detect suspicious movements in the home network.

One of the most studied areas in IoT-based smart homes is energy management, for reasons that electrical energy is limited, and its cost is ever-increasing. A common solution was an Energy Management System (EMS) [12] to monitor each device and acquire important data that will be used for data analytics. To test their proposed system, a small prototype focusing on HVAC (Heating, Ventilation and Air Conditioning) was built and tested. However, their EMS employed business intelligence and data analytics software that might not be suitable for small houses, i.e., with two to three rooms only and a few household occupants. Monitoring household conditions by reliably measuring and transmitting the smart sensor data was the focus of the work in [13]. The study discussed remote control of electric lamp and water heater in a non-obtrusive manner. On the other hand, the work found in [14] considered a home-based information-centric network that tackles data exchanges and network configurations. Study cases were used to assess the system's performance. Lastly, the Home Energy Management Systems presented in [15] has a central server that acts as the sole processor and means for sensing devices to exchange data. Communication among sensor nodes are limited and/or restricted.

In this work, we present a decentralized energy monitoring system, EnMonSys, that incorporates household and appliance energy profiling to determine energy anomaly, i.e., equipment/appliances that are operating beyond normal load. 
We focus on energy monitoring instead of management because our findings support that for a small and compact residential unit, there is little room for improvement in terms of reducing the average daily energy consumption. The main factor contributing to the increase or decrease of a small household electrical energy consumption cost is due to the change in season, addition or removal of household equipment, e.g., change from a non-inverter type refrigerator to an inverter type one, or change in household dweller. Decentralization of the proposed system is achieved by allowing sensor nodes to decide on its own what equipment must be turned ON/OFF without human intervention. The major contributions of this research study are summarized below.

1. A suitable energy monitoring system EnMonSys based on decentralized sensor placements in a compact smart home is proposed and experimented. EnMonSys logs individual sensor data and detects energy anomaly of an electrical equipment based on the household's standard energy consumption profile. To allow a decentralized control, each sensor node relies on predictive household movement to determine the utility of all controlled household devices and equipment, and further improve EnMonSys's energy anomaly detection.

2. Testing and evaluation of EnMonSys are done in a two-bedroom condominium unit with an area of 32 sqm holding four households.

This paper is organized as follows: Section II presents the smart home setup and deployment and interconnectivity of sensor nodes. The energy profiling procedure is also tackled here. Section III presents the preliminary experimental results and then introduces household profiling and predictive movement to improve EnMonSys's performance. Finally, Section IV summarizes the work and enumerates future directives of the study

\section{ENERGY MONITORING SYSTEM WITH ANOMALY DETECTION}

In this section, we discuss our proposed sensor node installation and energy monitoring system EnMonSys. EnMonSys relies on the standard energy profile of an equipment to detect energy anomaly, but later improved with the addition of household profile and predictive household movement schemes.

\subsection{Smart Home Floor Plan}

Figure 1 illustrates the floor plan of a 32 sqm condominium unit where four households live. The red squares represent the sensor/actuator nodes, representing the electrical and electronic devices being monitored. All sensor nodes are equipped with wireless Xbee radio transceivers to communicate and exchange data and commands, if any, from other sensor nodes and the Central Server (CS). (Please note that these links are all removed in the figure since all sensor nodes 0-7 have a direct wireless connection to the CS.) The yellow box ' $\mathrm{A}$ ' depicts the CS that is used only for data logging and user notification via cellular or WiFi transmission.

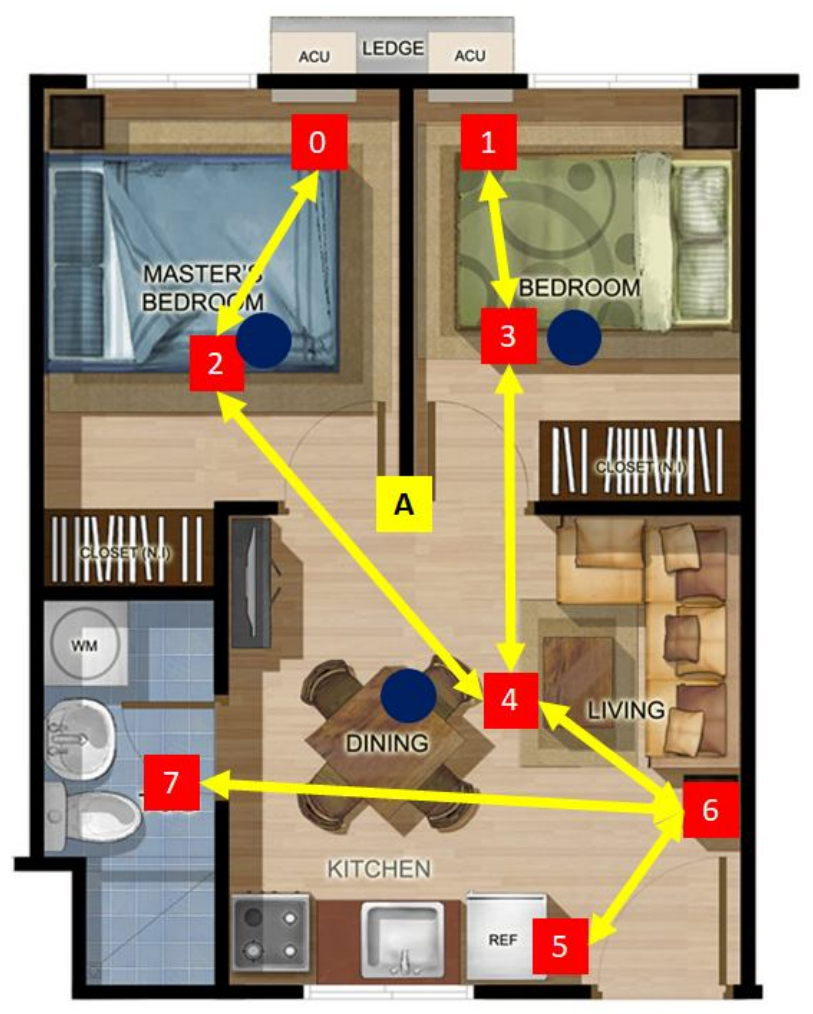

Figure 1: Household floor plan of a Smart Home. Numbered red boxes mean: (0-1) - Air Conditioning Units, (2-4) - Lights, (5) Refrigerator, (6) - Sockets/Electrical Outlets, and (7) Heater. The yellow arrows denote the node-to-node communication wireless link while the red shades illustrate the motion sensor's coverage area.

Motion sensors (blue circles) employ the PIR Motion Sensor HC-SR501 and are installed at the two bedrooms and living room. They are mounted at the ceilings and can detect human motion within a 120-degree area having a radius of five feet. However, for the living room, two motion sensors are utilized to have a 360-degree coverage area. The coverage areas of these motion sensors, except those in the bedrooms, are allowed to overlap and will be used in the predictive movement of household. Once activated, motion sensors' signals its corresponding sensor/actuator node, either sensor/actuator 2, 3, or 4. The turning ON/OFF of other electrical equipment ensues depending on the household's location.

Several wireless communication restrictions have been placed to reduce the links among all sensor/actuator nodes. These restrictions are depicted by the absence of a bi-directional yellow arrow between two sensor nodes. For example, air conditioning units (ACU) (red boxes 0 and 1 ) are not able to communicate due to their independence among themselves. 
Elmer R. Magsino, International Journal of Emerging Trends in Engineering Research, 7(8), August 2019, 151 - 156

Line-of-sight (LOS) path is ensured between sensor nodes to alleviate the multi-path fading problems. Such configuration may allow single or multi-hop communication among sensor nodes and bypasses the need to directly go to the CS for appropriate commands. However, during experimentations, only single hop communication is permitted for a simple network operation. Also, each sensor node can perform predictive movement with the aid of the motion sensors, effectively reducing the CS to a data logging center for the proposed EnMonSys.

\subsection{Energy Anomaly Detection}

The energy profiling for each electrical device is done to determine the normal operating energy consumption. Datasheets and device manuals are consulted for these. The electrical devices and equipment considered in the experiments are only those that can be automated and part of the daily routine of the household. Excluded in such assumption is the washing machine.

Table 1 shows the average energy profile of the household shown in Figure 1 obtained from a two-month testing period. It is assumed here that these are the normal operating conditions of each of the devices.

Table 1: Energy Consumption Profile of Household Devices/Equipment

\begin{tabular}{|l|c|c|l|}
\hline $\begin{array}{l}\text { Household } \\
\text { Device/ } \\
\text { Equipment }\end{array}$ & $\begin{array}{l}\text { Energy } \\
\text { Consumption } \\
\text { (in kWh) }\end{array}$ & $\begin{array}{l}\text { Ave. } \\
\text { Daily } \\
\text { Operating } \\
\text { Hours }\end{array}$ & Remarks \\
\hline $\begin{array}{l}\text { Light bulbs } \\
\text { (Living room) }\end{array}$ & 0.200 & 10 & $\begin{array}{l}\text { bulbs } \\
\text { Four }\end{array}$ \\
\hline $\begin{array}{l}\text { Light bulb } \\
\text { (Bedroom) }\end{array}$ & 0.025 & 5 & \\
\hline Heater & 2.0 & 1 & $\begin{array}{l}\text { At 39 } \\
\text { degrees }\end{array}$ \\
\hline ACU & 8.0 & 12 & $\begin{array}{l}\text { Two } \\
\text { units }\end{array}$ \\
\hline Refrigerator & 4.0 & 24 & \\
\hline $\begin{array}{l}\text { Microwave } \\
\text { Oven }\end{array}$ & 0.01336 & 0.0167 & \\
\hline Laptop & 0.2 & 4 & \\
\hline
\end{tabular}

Aside from experimentally obtaining the average normal energy consumption above, the following parameters are also empirically obtained for each device/equipment. The procedure to determine these values follow this setup. For an experimental period of $T_{\text {exp }}$, each device is turned ON while keeping the other equipment turned OFF. The average consumption is obtained by dividing the energy used by the trial period, in this case, Texp $=30$ mins. Energy usage is visually retrieved from the unit's electric meter display and is limited to the whole number consumption. The following findings are observed.
1. An ACU unit approximately has an average of 0.5 $\mathrm{kWh}$ change per degree of temperature change.

2. The heater approximately has an average of $1 \mathrm{kWh}$ change per degree of temperature change.

3. If the refrigerator door is left OPEN, the approximate average change is $1 \mathrm{kWh}$.

4. A laptop in sleep mode approximately consumes 0.05 $\mathrm{kWh}$ on the average. Using the energy profiling method only, energy anomaly is detected if the device goes above or below a certain preset threshold value for a prolonged time. This may be due to an unusual usage of an equipment/device, e.g., refrigerator door left opened, laptop normal operation going to sleep mode, busted light bulb, and ACU or Heater setting is changed. This is immediately seen in the vertical transition of the energy consumption of a device.

\section{EXPERIMENTAL RESULTS OF ENERGY HOUSEHOLD MONITORING}

In this section, experimental results coming from a two-month trial period are presented. We then present the household profile and predictive movement methods to improve EnMonSys.

\subsection{Preliminary Experimental Results}

In the first month of evaluation, controlled experiments are done in order to isolate the energy consumption of each device/equipment. Electrical equipment consuming energy that is greater than $1 \mathrm{kWh}$ are tested separately from those devices that consume less than $0.2 \mathrm{kWh}$.

Figure 2 illustrates a test scenario where the ACU's, refrigerator and heater are utilized together since these pieces of equipment are consuming more than $1 \mathrm{kWh}$ of energy. In the 'Total' plot, energy anomaly detection is determined by looking at the transitions (going up or going down).

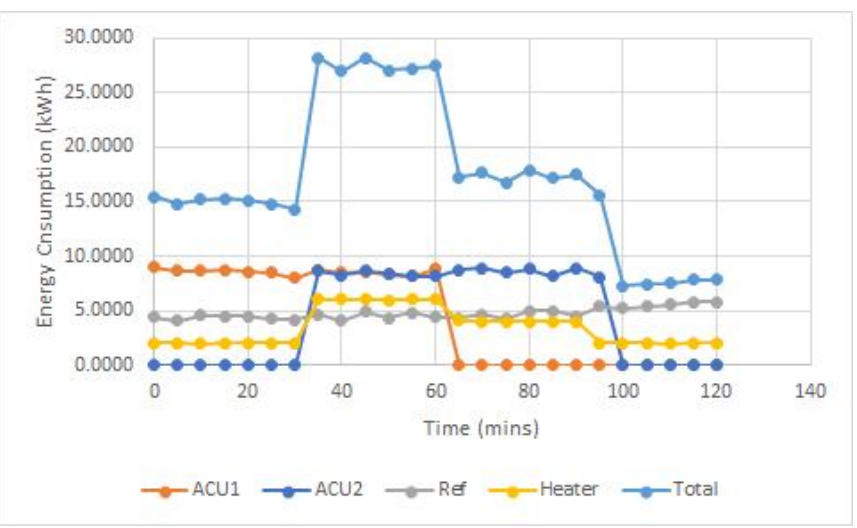

Figure 2: Average energy consumed by ACU's, refrigerator and heater.

In this scenario, the following has happened as shown by the 
individual plot of each equipment under test.

1. ACU 1 (Master's Bedroom) is turned ON only for the first hour.

2. ACU 2 (Bedroom) is turned $\mathrm{ON}$ after 30 minutes and turned OFF after one hour.

3. The refrigerator's door was intentionally opened after one and a half hour.

4. The heater is turned $\mathrm{ON}$ for 30 minutes at 39 degrees, then increased to 43 degrees for another thirty minutes. It is then decreased to 41 degrees for the next 30 minutes and finally set to its nominal temperature for the next 30 minutes.

On the other hand, Figure 3 illustrates the energy consumption of devices consuming energy below $0.2 \mathrm{kWh}$. Detecting of energy anomaly is also done by looking at the transitions, however, unlike the results depicted in the previous figure, identifying which is the cause of the rise or fall of energy consumption is difficult if the individual energy consumption are not shown since their usage is almost within the range of each other.

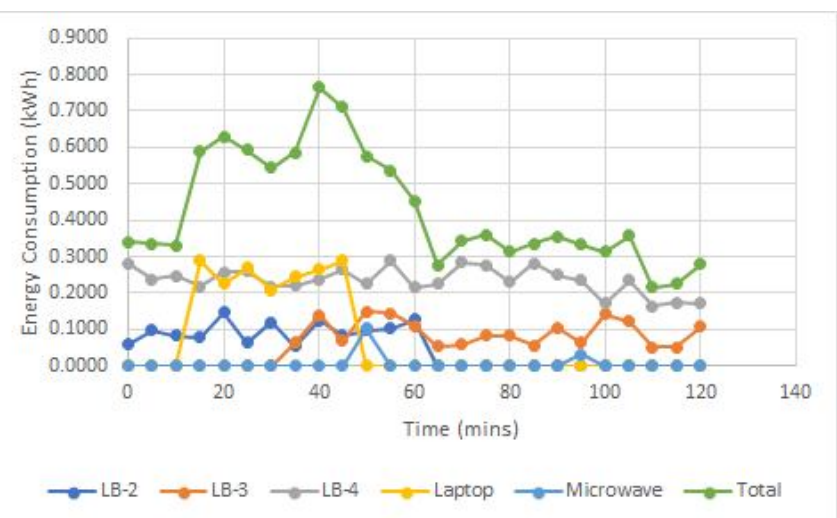

Figure 3: Average energy consumed by by light bulbs, laptop, and microwave oven.

The CS provides the user only the csv file and downloadable only at the end of the day. In our setup, we only download the daily data at 10 PM. Real-time energy monitoring is possible since sensor/actuator nodes log data every sampling time, however, such case does not give any valuable findings, thus, a csv downloadable file is chosen to allow the head of the household to analyze daily energy usage.

\subsection{Enhancing the Energy Anomaly Detection Scheme via Household Profile}

Our preliminary results provide insights on how devices and equipment behave during operation. The experiments above highlight that the transition in the logged consumption is a sign of an energy anomaly, wanted or unwanted. To determine which is not deliberately done, we enhance the energy anomaly detection by incorporating the household energy profile, as shown in Table 2.
Given Table 2 and the logged data of each sensor, the CS correlates which vertical transition is deliberately done. In the experiments, during the first trial month, we were only able to determine $75 \%$ of the energy anomaly occurrences correctly. In the next month, we were able to increase it to approximately $81 \%$. It is suspected that as the actions of the households become more routinely, this anomaly detection will increase because the household profile will also be updated.

Table 2: Household Profile of using Electrical Equipment/Device during July and August

\begin{tabular}{|l|l|}
\hline Equipment/Device & Time of Operation \\
\hline ACU 1 (Master's Bedroom) & 7 PM - 7 AM \\
\hline ACU 2 (Bedroom) & 7 PM - 7 AM \\
\hline Refrigerator & 24 hours \\
\hline \multirow{2}{*}{ Heater } & 8 - 9:00 AM \\
& 7 - 8:00 PM \\
\hline Light bulbs & No fixed schedule \\
\hline \multirow{2}{*}{ Laptop } & 9 - 11:00 AM \\
\hline Microwave & 9 - 11:00 PM \\
\hline
\end{tabular}

\subsection{Predictive Movement of Households to Improve EnMonSys}

To this point, we have not yet incorporated the data coming from the motion sensor and have not introduced an automated procedure. Since the testing venue is a $32 \mathrm{sqm}$ condominium unit only, we exploited its small area to come up with a predictive household movement shown in Table 3. The idea of incorporating predictive household movement is to further introduce another criterion on how to detect energy anomaly. Each sensor/actuator node implements Tables 2-3 via lookup table.

Table 3: Predictive Household Movement Scenarios. ' 1 ' denotes possible destination, else ' $\mathrm{X}$ '.

\begin{tabular}{|l|c|c|c|c|}
\hline & \multicolumn{4}{|c|}{ Destination } \\
\hline Source & $\begin{array}{l}\text { Living } \\
\text { Room }\end{array}$ & $\begin{array}{l}\text { Master's } \\
\text { Bedroom }\end{array}$ & Bedroom & Toilet \\
\hline $\begin{array}{l}\text { Living } \\
\text { Room }\end{array}$ & $\mathrm{X}$ & 1 & 1 & 1 \\
\hline $\begin{array}{l}\text { Master's } \\
\text { Bedroom }\end{array}$ & 1 & $\mathrm{X}$ & $\mathrm{X}$ & $\mathrm{X}$ \\
\hline Bedroom & 1 & $\mathrm{X}$ & $\mathrm{X}$ & $\mathrm{X}$ \\
\hline Toilet & 1 & $\mathrm{X}$ & $\mathrm{X}$ & $\mathrm{X}$ \\
\hline
\end{tabular}




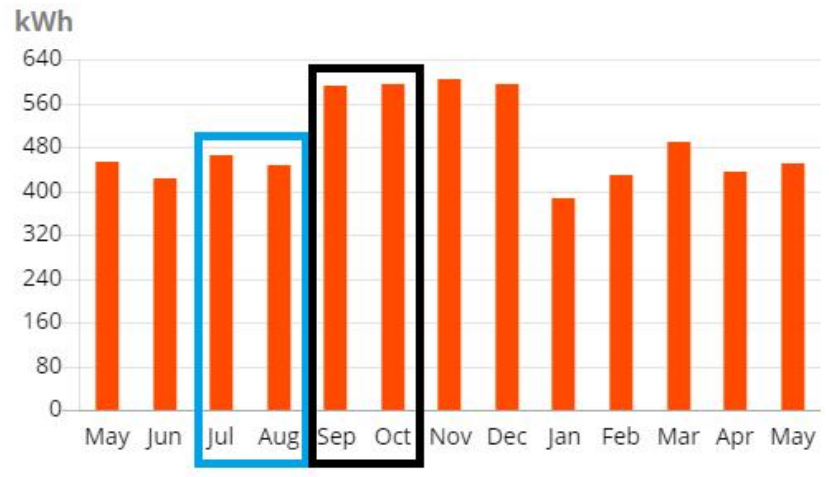

Figure 4: Monthly energy consumption.

Figure 4 showcases the performance of EnMonSys for the test months of August and September 2018. We notice that the energy consumption for these two months are the same as July and October 2018, respectively. This means that the energy consumption change from August to September 2018 is brought about only by the change in weather. August 2018 is a much cooler month when compared to September of 2018 because of the typhoon season. Therefore, the household profile use of the ACU's was increased, effectively, bringing the energy consumption up. This is given in Table 4.

Given Figure 4, we observe that without the change in household and addition of any electrical equipment, EnMonSys can monitor the energy consumption without really managing the household electrical appliances. This is opposed to buildings with energy management system whose occupants are mostly employees.

Finally, Table 5 compares the results of the three methods used to detect energy anomaly. EnMonSys combines the use of both energy and household profiles and the predictive household movement for monitoring the consumption and detecting the anomaly when the refrigerator door was left opened. More so, EnMonSys can control the lights when there is occupancy in a certain room. This is an automation added convenience to the household which is also a feature in most building EMS.

Table 5: Comparison of Energy Anomaly Detection given the Three Methods

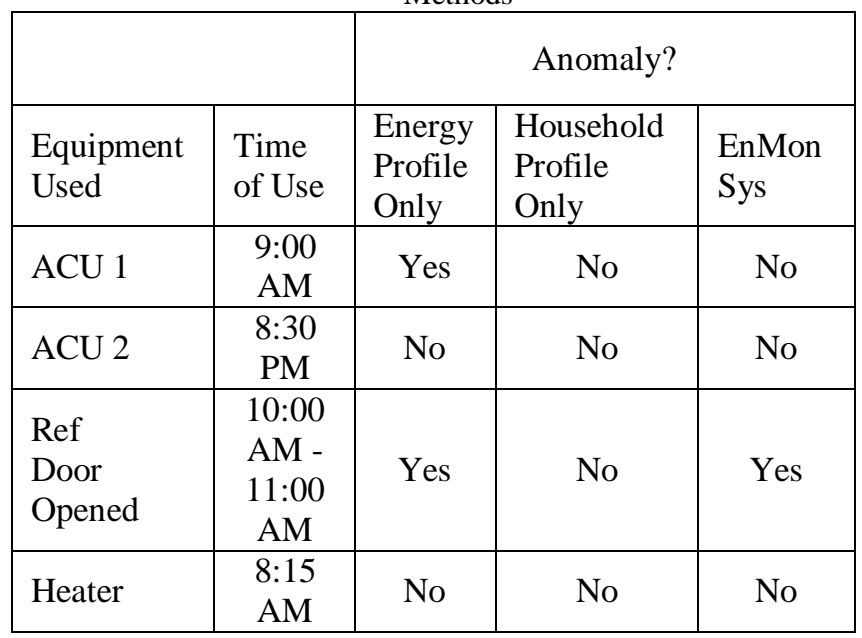

\section{CONCLUSION AND FUTURE WORK}

In this study, a small household energy monitoring system, EnMonSys, has been designed and implemented to monitor the utility of household appliances. We have incorporated household profile and predictive movement to the energy profile to increase the detection of energy wastage. EnMonSys has also provided automatic actuation of room lights for user convenience and as a manner of visually knowing that EnMonSys is actively monitoring the household environment. As the household activity profile is updated and correlated with the movements of the dwellers, the anomaly detection of EnMonSys increases, while at the same time providing living convenience and comfort. In the future, when the bedroom doors are closed, the experimental setup is just reduced to three smaller and independent testbeds, which will require a non-line-of-sight indoor positioning system [16] if the events in these three rooms are to be correlated. If small households have such energy monitoring systems, these consumption data can be used for electrical load forecasting [17]. Finally, PID [18] and fuzzy logic controllers [19] for complex and nonlinear systems such as unmanned aerial vehicles and connected vehicles can be incorporated to achieve system performance and target operating specifications.

\section{REFERENCES}

[1]A. Whitmore, A. Agarwal, and L. Da Xu, "The internet of things: a survey of topics and trends," Information Systems Frontiers, vol. 17, no. 2, pp. 261-274, 2015. https://doi.org/10.1007/s10796-014-9489-2

[2] L. Da Xu, W. He, and $\mathrm{S}$. Li, "Internet of things in industries: A survey," IEEE Transactions on industrial informatics, vol. 10, no. 4, pp. 2233-2243, 2014. https://doi.org/10.1109/TII.2014.2300753

[3]E. R. Magsino and I. W.-H. Ho, "Roadside unit allocation for fog-based information sharing in vehicular networks," in Proceedings of the 1st ACM International Workshop on Smart Cities and Fog Computing. ACM, 2018, pp. 7-12. https://doi.org/10.1145/3277893.3277897

[4]P. Gope and T. Hwang, "Bsn-care: A secure iot-based modern healthcare system using body sensor network," IEEE sensors journal, vol. 16, no. 5, pp. 1368-1376, 2015. https://doi.org/10.1109/JSEN.2015.2502401

[5]L. Atzori, A. Iera, and G. Morabito, "The internet of things: A survey," Computer networks, vol. 54, no. 15, pp. 2787-2805, 2010. https://doi.org/10.1016/j.comnet.2010.05.010

[6] A. M. D. Celebre, A. Z. D. Dubouzet, I. B. A. Medina, A. N. M. Surposa, and R. C. Gustilo, "Home automation using raspberry pi through siri enabled mobile devices," in 2015 International Conference on Humanoid, Nanotechnology, Information Technology, Communication and Control, Environment and Management (HNICEM). 
IEEE, 2015, pp. 1-6. https://doi.org/10.1109/HNICEM.2015.7393270

[7]A. P. Plageras, K. E. Psannis, C. Stergiou, H. Wang, and B. B. Gupta, "Efficient iot-based sensor big data collection-processing and analysis in smart buildings," Future Generation Computer Systems, vol. 82, pp. 349-357, 2018. https://doi.org/10.1016/j.future.2017.09.082

[8]D. Kwon, M. R. Hodkiewicz, J. Fan, T. Shibutani, and M. G. Pecht, "Iot-based prognostics and systems health management for industrial applications," IEEE Access, vol. 4, pp. 3659-3670, 2016.

[9]B. L. R. Stojkoska and K. V. Trivodaliev, "A review of internet of things for smart home: Challenges and solutions," Journal of Cleaner Production, vol. 140, pp. 1454-1464, 2017.

[10] R. K. Kodali, V. Jain, S. Bose, and L. Boppana, "Iot based smart security and home automation system," in 2016 international conference on computing, communication and automation (ICCCA). IEEE, 2016, pp. 1286-1289.

[11] V. Sivaraman, H. H. Gharakheili, A. Vishwanath, R. Boreli, and O. Mehani, "Network-level security and privacy control for smart-home iot devices," in 2015 IEEE 11th International conference on wireless and mobile computing, networking and communications (WiMob). IEEE, 2015, pp. 163-167. https://doi.org/10.1109/WiMOB.2015.7347956

[12] A.-R. Al-Ali, I. A. Zualkernan, M. Rashid, R. Gupta, and M. Alikarar, "A smart home energy management system using iot and big data analytics approach," IEEE Transactions on Consumer Electronics, vol. 63, no. 4, pp. 426-434, 2017.

[13] S. D. T. Kelly, N. K. Suryadevara, and S. C. Mukhopadhyay, "Towards the implementation of iot for environmental condition monitoring in homes," IEEE sensors journal, vol. 13, no. 10, pp. 3846-3853, 2013.

[14] M. Amadeo, C. Campolo, A. Iera, and A. Molinaro, "Information centric networking in iot scenarios: The case of a smart home," in 2015 IEEE international conference on communications (ICC). IEEE, 2015, pp. 648-653.

[15]Z. Zhao, W. C. Lee, Y. Shin, and K.-B. Song, “An optimal power scheduling method for demand response in home energy management system," IEEE Transactions on Smart Grid, vol. 4, no. 3, pp. 1391-1400, 2013. https://doi.org/10.1109/TSG.2013.2251018

[16]E. R. Magsino, I. W.-H. Ho, and Z. Situ, "The effects of dynamic environment on channel frequency response-based indoor positioning," in 2017 IEEE 28th Annual International Symposium on Personal, Indoor, and Mobile Radio Communications (PIMRC). IEEE, 2017, pp. 1-6.

[17]L. F. Torrizo and A. D. Africa, "Next-hour electrical load forecasting using an artificial neural network: Applicability in the Philippines,'
International Journal of Advanced Trends in Computer Science and Engineering, vol. 8, no. 3, pp. 831-835, 2019. https://doi.org/10.30534/ijatcse/2019/77832019

[18] G. P. T. Mayuga and E. R. Magsino, "Adaptive cruise control employing taillight tracking for a platoon of autonomous vehicles," International Journal of Advanced Trends in Computer Science and Engineering, vol. 8, no. 3, pp. 640-645, 2019. https://doi.org/10.30534/ijatcse/2019/48832019

[19] E. R. Magsino, K. Obias, J. P. Samarista, M. F. Say, and J. A. Tan, "A redundant flight recovery system implementation during an octocopter failure," in 2016 IEEE Region 10 Conference (TENCON). IEEE, 2016, pp. 1825-1828. https://doi.org/10.1109/TENCON.2016.7848336 